\title{
Esophageal self-expandable metal stent for an anastomotic colorectal stricture
}

A 49 year-old woman with colonic inertia required total colectomy with ileorectal anastomosis in November 2009. She subsequently developed partial bowel obstruction requiring multiple balloon dilations of a pinpoint anastomotic stricture. A repeat endoscopy was performed and the stricture was first dilated up to $10 \mathrm{~mm}$ with a CRE balloon dilator (Boston Scientific, Natick, Massachusetts, USA; Fig. 1 a, b).

A fully covered, metal, $18 \times 80-\mathrm{mm}$ esophageal Bonastent (Standard Sci Tech Inc., Seoul, South Korea) was then deployed across the stricture ( $\nabla$ Fig. $\mathbf{1}$ c, d) under fluoroscopic guidance ( Fig. 2). The patient tolerated the procedure well, without any complications.

She was continuing to do extremely well when she returned for follow-up visits 1 month and 3 months later, with a barium enema showing a patent stent. She has declined to have the stent removed because she is finally symptom free.

This case is unusual in that an esophageal stent was used to treat a colonic stricture. In patients with advanced malignancies, colonic stenting can provide palliative care by relieving acute colonic obstruction [1]. More recently, self-expanding metal stents (SEMS) have been placed in patients with benign colorectal disease [2]. The use of SEMS may be associated with a longer lasting dilatation and a lower rate of recurrence in cases of anastomotic stricture [2].

The majority of the previously reported cases have used colonic stents, either covered or uncovered [3]. However, for rectosigmoid lesions, esophageal stents may be another useful option because they are covered and the relatively short distance from the anus offers greater mechanical advantage in terms of manipulation of the stents $[3,4]$. One clinical trial that compared the migration rates of several stents in malignant colonic strictures reported that the Bonastent had the lowest rate of migration. This was attributed to its larger diameter and flared ends [4].
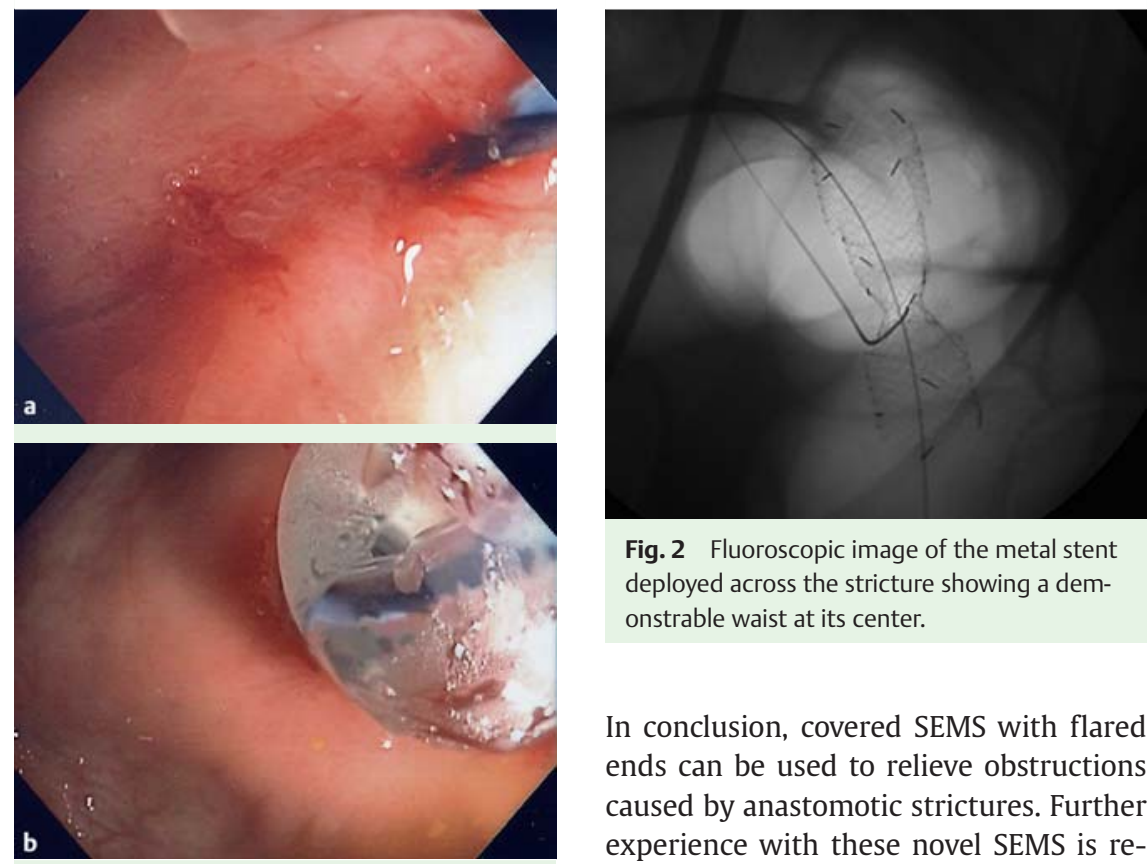

Fig. 2 Fluoroscopic image of the metal stent deployed across the stricture showing a demonstrable waist at its center.

In conclusion, covered SEMS with flared ends can be used to relieve obstructions caused by anastomotic strictures. Further experience with these novel SEMS is required before they can be recommended for more generalized use.
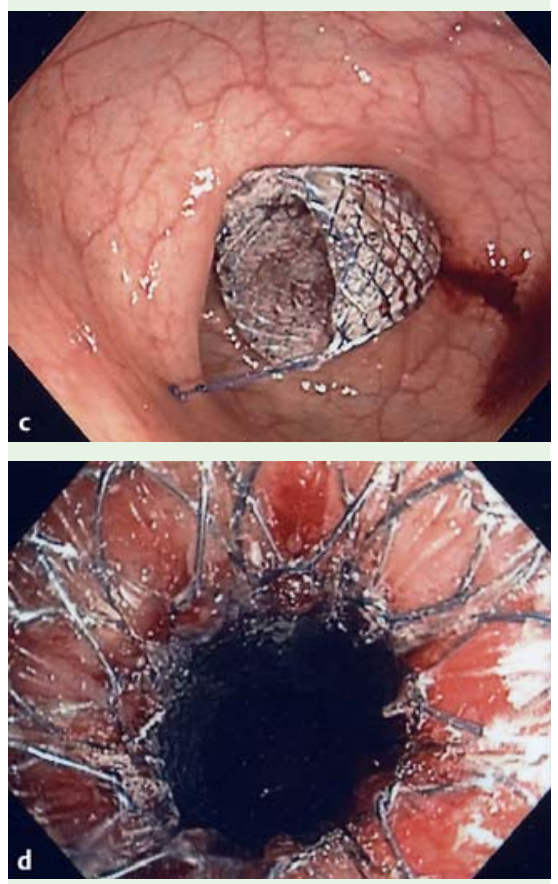

A. Mahajan', M. Rothermich²,

M. Gaidhane ${ }^{3}$, M. Kahaleh ${ }^{3}$

1 Department of Gastroenterology and Hepatology, John Hopkins Medical Center, Baltimore, Maryland, USA

2 University of Virginia School of Medicine, Charlottesville, Virginia, USA

3 Division of Gastroenterology \& Hepatology, Department of Medicine, Weill Cornell Medical College, New York, New York, USA

Competing interests: Dr Kahaleh has retific, Mi Tech, Fuji, and Pentax. He is a consultant for Boston Scientific and Xlumina.
Fig. 1 Endoscopic view showing: a the wire placed across the anastomotic stricture; b the wire-guided balloon dilating the stricture; c the esophageal, covered, metal stent deployed across the stricture; and $\mathbf{d}$ a close-up of the stent inner lumen post deployment. 


\section{References}

1 Targownik LE, Spiegel BM, Sack J et al. Colonic stent vs. emergency surgery for management of acute left-sided malignant colonic obstruction: a decision analysis. Gastrointest Endosc 2004; 60: 865 - 874

2 Forshaw MJ, Sankararajah D, Stewart M, Parker MC. Self-expanding metallic stents in the treatment of benign colorectal disease: indications and outcomes. Colorectal Dis 2005; 8: $102-111$
3 Bonin EA, Baron TH. Update on the indications and use of colonic stents. Curr Gastroenterol Rep 2010; 12: 374-382

4 Park JK, Lee MS, Ko BM et al. Outcome of palliative self-expanding metal stent placement in malignant colorectal obstruction according to stent type and manufacturer. Surg Endosc 2011; 25: 1293-1299

\section{Bibliography}

DOI $10.1055 / \mathrm{s}-0030-1256959$

Endoscopy 2011; 43: E415-E416

(c) Georg Thieme Verlag KG Stuttgart · New York . ISSN 0013-726X

\section{Corresponding author}

M. Kahaleh, MD

Advanced Endoscopy

Division of Gastroenterology \& Hepatology

Department of Medicine

1305 York Avenue, 4th Floor

New York, New York 10021

USA

Fax: +1-646-962-0110

mkahaleh@gmail.com 